\title{
Prevalence, characteristics and maternal risk factors of small for gestational age fetuses in a tertiary care center from Kerala
}

\author{
Subhadra Subramanian, Remadevi S, Karthik Subramaniam \\ Corresponding author: Dr Karthik Subramaniam, Department of Endocrinology, Silverline \\ Hospital, Kochi, Kerala, India; Email - smkarthik.kmc@gmail.com
}

Distributed under Attribution-Non Commercial - Share Alike 4.0 International (CC BY-NC-SA 4.0)

\begin{abstract}
Objectives: This study was conducted to determine the prevalence and to understand the maternal characteristics and risk factors of small for gestational age (SGA) fetuses among booked antenatal mothers in the third trimester in a tertiary care hospital. Methods: 250 consecutive booked and singleton mothers at or above 28 weeks gestation were screened for SGA. Those who were diagnosed so, were matched 1:2 ratio with age matched controls and compared. Results: The prevalence of SGA was $13.6 \%$ (95\% CI 9.4-17.8). Fifty-three percent were in the age group of 20-24 years, $68 \%$ were primigravida and $75 \%$ of multigravida women had previous history of SGA child. Mothers of SGA fetuses had median BMI of $22.4 \mathrm{~kg} / \mathrm{m}^{2}$ and gained $8 \mathrm{~kg}$ in pregnancy. Each kilogram gain in pre-pregnancy weight reduces the risk of having SGA fetus by $0.8 \%$. Each earlier week of delivery increased the risk of LBW by $20 \%$. Each gram of low hemoglobin increased the risk of having SGA fetus by 7.6\%. Mothers with previous history of SGA had odds of 36 times to have SGA fetus in the current pregnancy. Conclusions: Apart from knowing the prevalence, this study also sheds light into fact that several social, educational and nutritional factors play a role in causation of a SGA fetus. Health policies aimed in improving adolescent health and education could be one of the primary preventive strategies.
\end{abstract}

Keywords: Fetal growth restriction, intrauterine growth restriction, South India.

Intrauterine growth restriction (IUGR) or fetal growth restriction (FGR) is a common complication of pregnancy leading to multiple adverse perinatal outcomes. The prevalence of FGR is variable but grossly affects 5-10\% of uncomplicated gestations and up to $25 \%$ in high risk gestation. Also it is the second most common cause of perinatal mortality ${ }^{1}$. FGR babies have an increased risk for perinatal mortality, complications of prematurity and birth adaptation. On a long-term basis, they have an increased risk for developing short stature, cognitive delay with decreased academic achievement and increased risk of neurologic disorders, including cerebral palsy ${ }^{2}$. FGR is now proven to be associated with increased rates of coronary heart disease, stroke, hypertension, PCOS in women and type 2 diabetes ${ }^{3}$. Hence, it is important to diagnose and mitigate this condition, as it would lead to substantial burden to the society on long-term basis.

Small for gestational age (SGA) is not synonymous with FGR but due to ease in diagnosis of SGA, we refer further discussions strictly to SGA. The prevalence of SGA fetuses is variable across the globe. In India, the estimates were up to $47 \%$ with high regional variation. Latest and largest study

Received: $23^{\text {rd }}$ February 2020. Accepted: $2^{\text {nd }}$ April 2020.

Subramanian S, Remadevi S, Subramaniam K. Prevalence, characteristics and maternal risk factors of small for gestational age fetuses in a tertiary care center from Kerala. The New Indian Journal of OBGYN. 2020; 7(1): 76-81. 
from south India comes from the state of Tamil Nadu, where the prevalence of SGA among about 37000 deliveries was $8.4 \%{ }^{4}$. From the previous discussion; it is clearly evident that SGA has a huge impact on the future health of the individual. In order to prevent SGA, maternal correctable risk factors have to be established. Since both these statesKerala and Tamil Nadu fare almost equally in health parameters, it is prudent to even compare the incidence of SGA. In order to tailor the health policies for the state, a proper investigation for the prevalence is required - this was the primary reason for the conduct of the study.

Maternal, fetal and placental risk factors lead to a 'stressed' fetus with high levels of inflammatory markers. Several markers like interleukin-6, tumour necrosis factor $\alpha$, $\mathrm{C}$ reactive protein, myeloperoxidase and adiponectin levels have been demonstrated to be significantly high in cord blood samples 5,6 . Hence, this study was conducted to determine the prevalence of SGA fetuses among booked antenatal mothers in the third trimester in a tertiary care hospital.

\section{Materials and methods}

This was a hospital based cross sectional study with a nested case control comparison. The study was conducted from May 2018 to December 2018 in the outpatient department of Obstetrics and Gynecology. For the prevalence study, 250 consecutive booked and singleton antenatal mothers at or above 28 weeks were enrolled after getting voluntary consent. Twin or multiple gestation pregnancies, fetuses diagnosed with congenital anomalies and mothers referred from other hospitals were excluded. All antenatal mothers enrolled in the study were screened by ultrasonogram as per protocol. Antenatal mothers having estimated fetal weight less than the $10^{\text {th }}$ percentile for their gestational age (reference for estimated fetal weight was taken from a study ${ }^{7}$ were diagnosed to have SGA fetus and were enrolled for the second part of the study. The definitions of different variables are available in supplementary appendix.

All antenatal mothers with fetus diagnosed to have SGA by definition from the prevalence study were taken as cases. Mothers with AGA fetus were randomly chosen by simple random numbers from the remaining sample and were taken as controls for comparison (cases: controls $=1: 2$ ). For the case-control study, details of antenatal mothers with SGA residential, nutritional, medical and socioeconomic - were noted. Anthropometry and complete examination was performed. Biochemical tests - hemoglobin, TSH, glucose challenge test and CRP were performed. AFI levels and placental insufficiency indices in USG; PAPP-A and $\beta$ hCG levels (when available) were recorded. For the controls (who were selected by random numbers from the prevalence cohort), all the above said details were recorded except for CRP.

The antenatal mothers labeled cases were followed up till confinement. Additional nutritional supplements given to them were recorded. Maternal outcomes in terms of mode of delivery and timing of delivery were recorded. Immediate neonatal outcomes in terms of APGAR score, admission in NICU and duration of hospital stay were recorded.

Statistical analysis: Expected proportion of SGA as per previous study from CMC Vellore was $8.4 \%{ }^{4}$. With total width of confidence interval (W) being 0.1 and confidence level (CL) of $99 \%$, the sample size was $204^{8}$. Continuous variables were expressed as mean \pm standard deviation (SD) or median with interquartile range whichever is appropriate. Frequencies and percentages were used for categorical data. Distribution of variables was checked for normality with Shapiro-Wilk test. Pearson's Chi-square test was used to assess differences in groups for categorical data. Normative data was compared by student-t test and non-normative by Mann Whitney U test. Spearman's correlation was done to assess the relation between fetal birth weight and maternal parameters. Variables showing significant correlation and other relevant parameters were entered into stepwise backward binary logistic regression to calculate odds ratio. Data was analyzed by the Statistical Package for the Social Sciences software (version 23.0, IBM, SPSS Statistics). A p value of $<0.05$ was considered statistically significant.

\section{Results}

Description of SGA pregnancies: The prevalence of SGA in our study was $13.6 \%(95 \%$ CI $9.4-17.8)$ in 250 consecutive antenatal mothers. Of 34 SGA fetuses, 33 had birth weight less than $2.5 \mathrm{~kg}$. None of the 68 randomly selected controls had low birth weight. Fifty-three percent of mothers were between $20-24$ years of age (Figure 1). About $60 \%$ were graduates and $18 \%$ were employed. Half of them were residing in urban areas. Except for one, all mothers conceived naturally. Majority was primigravida (68\%) and there was inter-pregnancy spacing of $>3$ years in $75 \%$ of multigravida mothers. Previous history of SGA was present in $75 \%$ of multigravida women. About $25 \%$ had chronic medical disorders prior to pregnancy (Figure 2). Sixty-two percent were already on nutritional supplements prior to consultation. While the timing of delivery, birth weight and 
Table 1: Antenatal details of cases and controls

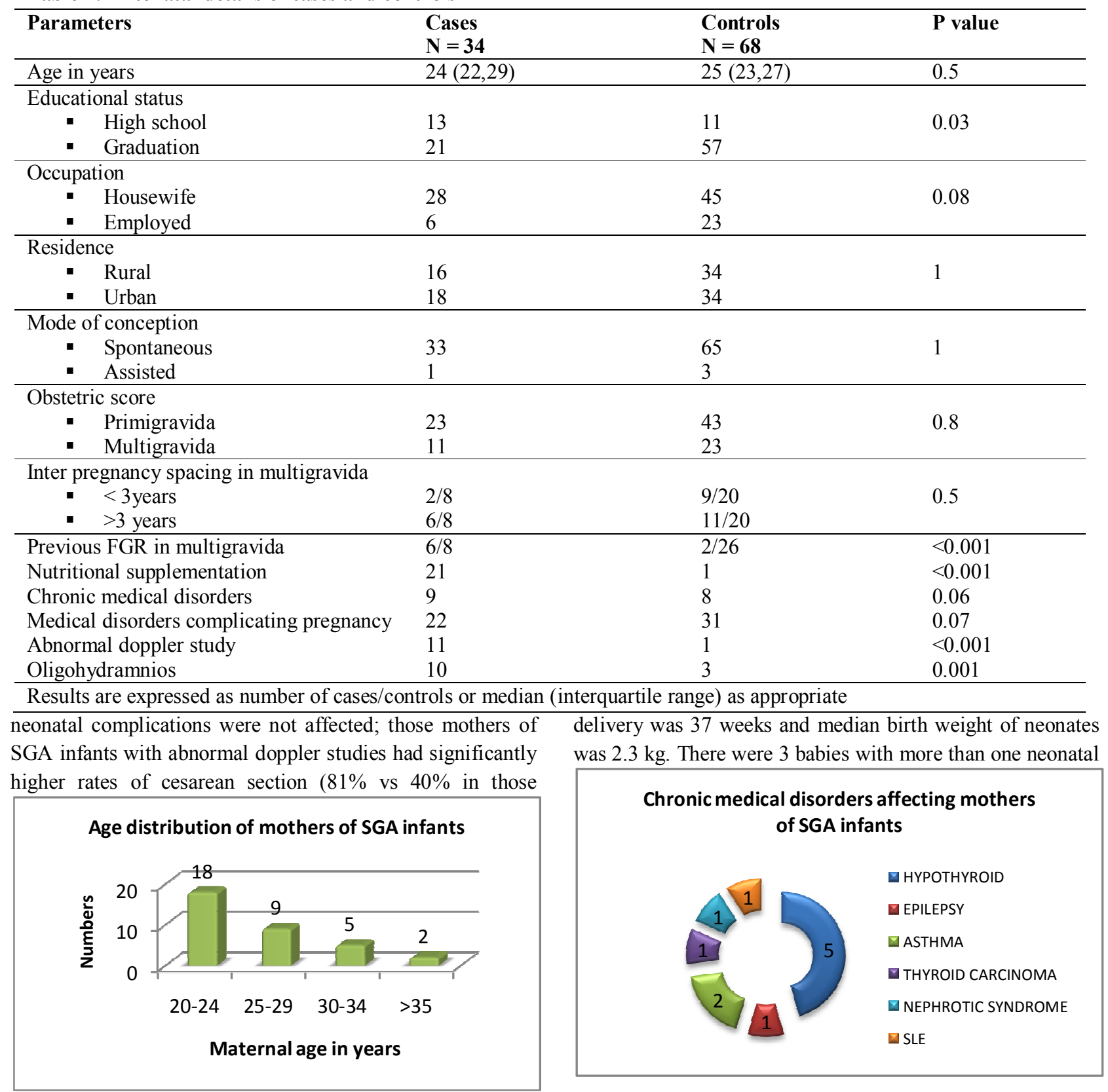

Figure 1: Age distribution of mothers of SGA infants

without abnormal doppler). Anthropometrically, mothers had median BMI of $22.4 \mathrm{~kg} / \mathrm{m}^{2}$ and gained $8 \mathrm{~kg}$ in pregnancy. Details of mode of delivery of the mothers have been elaborated in figure 3. Median gestational age at the time of
Figure 2: Chronic medical disorders affecting mothers of SGA infants

complication. Two babies required ventilator support and one baby was delivered after intrauterine death. Comparison of mothers of SGA infants with controls: The median age was not different between cases and controls (Table 1). Proportionately, $84 \%$ of controls were graduate or higher in 


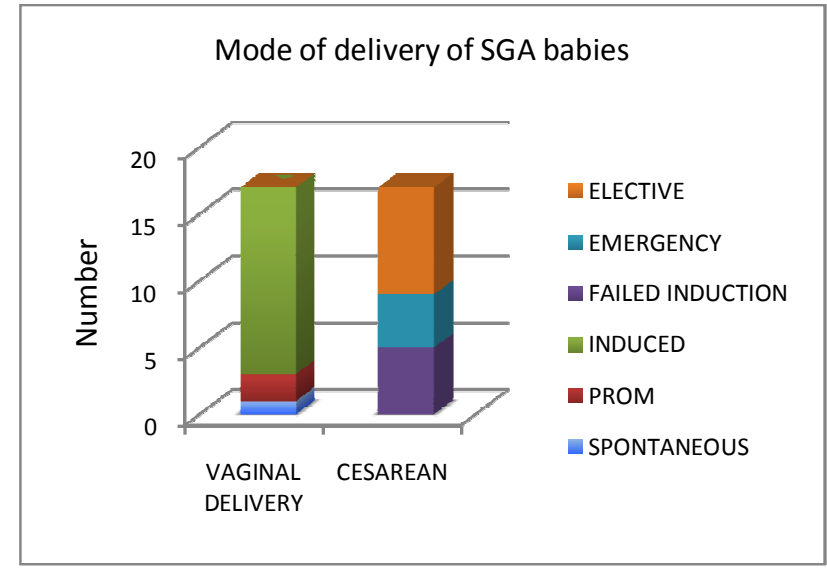

Figure 3: Mode of delivery of SGA babies

qualification whereas it was only $61 \%$ in cases. There was significantly higher number of mothers in SGA group with previous SGA births. While 21 of 34 in SGA group were on nutritional supplementation already, only 1 of 68 in control group was on supplementation. There was significant difference in pre-pregnancy weight between two groups (Table 2). Cases were significantly more stunted than two groups. A significant weight gain difference of $3 \mathrm{~kg}$ was present between controls and cases. Median hemoglobin level was lower in cases. With respect to perinatal outcomes, the timing of delivery was significantly earlier in cases median of 37 weeks as compared to 39 weeks in controls. As expected, number of cesarean section was higher in cases $55 \%$.

Correlation and regression: One of the hypotheses that, maternal inflammatory markers would be related to fetal weight was not found to be true. However, there were correlations between maternal weight $(\mathrm{r}=+0.27, \mathrm{p}=0.006)$, weight gain during pregnancy $(\mathrm{r}=+0.36, \mathrm{p}<0.001)$ and maternal height $(\mathrm{r}=+0.37, \mathrm{p}<0.001)$ with birth weight. When analyzed with univariate general linear model, the adjusted $\mathrm{R}^{2}$ was 0.65 , meaning that the predicted estimate was very significant. Each kilogram gain in pre-pregnancy weight reduces the risk of having SGA fetus by $0.8 \%$. Time of delivery has been very well correlated with birth weight. In our study, each earlier week of delivery increased the risk of LBW by $20 \%$. Each gram of low hemoglobin increased the risk of having SGA fetus by $7.6 \%$. Babies born out of

Table 2: Anthropometric, biochemical and perinatal details of cases and controls

\begin{tabular}{llll}
\hline Parameters & Cases & Controls & P value \\
& $\mathbf{N}=\mathbf{3 4}$ & $\mathbf{N = 6 8}$ & 0.001 \\
\hline Prepregnancy weight in $\mathrm{Kg}$ & $50.5(42,59)$ & $58.5(50.3,67)$ & $<0.001$ \\
Height in cm & $151 \pm 6$ & $157 \pm 6$ & 0.07 \\
BMI in Kg/m & $22.4(18.5,25.5)$ & $23.9(20.9,26.7)$ & $<0.001$ \\
Weight gain in pregnancy in $\mathrm{Kg}$ & $8(5.7,11)$ & $11(9.3,13)$ & 0.04 \\
Hemoglobin in g/dL & $11.6 \pm 1.2$ & $12.1 \pm 1$ & 0.8 \\
TSH in mIU/mL & $2.2(1.4,3.5)$ & $2.2(1.4,3.6)$ & 0.6 \\
Glucose challenge test in mg/dL & $124(112,138)$ & $127(111,157)$ & 0.9 \\
\hline PAPP-A in MoM & $1.03(0.67,1.31)$ & $0.94(0.67,1.62)$ & 0.07 \\
& $\mathrm{~N}=22$ & $\mathrm{~N}=34$ & $\mathrm{NA}$ \\
\hline Free $\beta$ hCG in MoM & $1.1(0.8,1.6)$ & $0.7(0.4,1.4)$ & $<0.001$ \\
CRP in mg/L & $5(3.4,7.4)$ & $\mathrm{NA}$ & \\
Timing of delivery in weeks & $37(36.5,37.7)$ & $39(38,39.4)$ & 0.006 \\
\hline Mode of delivery & & & \\
$\quad$ - Vaginal $\quad$ LSCS & 16 & 52 & 0.6 \\
\hline Gender of baby (female) & 20 & 16 & $<0.001$ \\
Birth weight in Kg & $2.3(2.1,2.4)$ & 37 & $<0.001$ \\
APGAR score & $9(8,9)$ & $3.2(3,3.5)$ & $<0.001$ \\
NICU stay in days & $2(0,4)$ & $9(9,9)$ & $0(0,0)$ \\
\hline Results are expressed & & & \\
\hline
\end{tabular}

Results are expressed as number of cases/controls, median (interquartile range) or mean \pm standard deviation as appropriate.

Abbreviations: BMI - body mass index, TSH - thyroid stimulating hormone, PAPP-A - pregnancy associated plasma protein - A, MoM - multiples of median, hCG - human chorionic gonadotropin, CRP - C reactive protein, LSCS - lower segment cesarean section, NICU - neonatal intensive care unit

controls. As a result of these changes, BMI, a fraction vaginal delivery had 14\% reduced risk of LBW. In logistic involving both height and weight was not different between regression of multigravida women, it was seen that mothers 
with previous history of SGA had odds of 36 times to have SGA fetus in the current pregnancy.

\section{Discussion}

The prevalence of SGA fetuses in the current study was $13.6 \%$. This figure closely resembled that obtained in a large retrospective study comprising about $>36000$ deliveries done in a tertiary care center in Tamil Nadu. However, SGA was defined based on birth weight $<10^{\text {th }}$ centile in that study ${ }^{4}$. The prevalence was $11.4 \%$ in 1996 and $8.4 \%$ in 2010 . Considering the same health related indices, socioeconomic factors and almost similar ethnicity, these results were comparable.

Considering the national average of SGA at birth being up to $47 \%$, it is important to know the proportion during gestation in order to allocate health resources. Detection of SGA fetus gives an ample opportunity for the obstetrician to anticipate and prepare adequately for perinatal complications 1. However, until recently there were no specific interventions to treat a SGA fetus so as to normalize its weight by birth. Nutritional supplementation/ modification in 4665 women from 13 trials did increase the birth weight minimally and reduce the SGA infants modestly (RR 0.68, $95 \%$ CI $0.56-0.84)$. Paradoxically, there was no additional benefit in nutritionally deprived section ${ }^{9}$. In another metaanalysis comprising $>137000$ pregnancies from low to middle-income countries, multiple micronutrient supplementation resulted in reduction of SGA neonates (RR 0.92 , 95\% CI 0.86 - 0.98). There was no significance difference when micronutrients were compared with iron/folate supplementation ${ }^{10}$. These studies again confirm that as of now there are few evidences to support iron and folate supplementation for treating SGA fetus to prevent LBW.

Social factors had great impact in deciding the outcome of fetal weight. In our study, educational status played a major role. As it can be appreciated from the final predictive model equation, educational status of high school was tending towards significance to predict the risk of SGA fetus. The green top guideline from NICE does not recognize this as a risk factor probably because in such a developed country educational status may not be a risk factor. But, in developing country such as India, through our study, we propose to include educational status as one of the risk factor of determination of SGA fetus in third trimester. Though it is grossly emphasized that education of girls is important for improvement of society, there is objective evidence from our study that empowering women with higher education pays off by improving the health of future generation.

Two-thirds of mothers of SGA infants were primigravida and half were between 20-24 years. These findings again vouch for improving the general health status of girls from birth so that they are healthy during reproductive years. Though elderly age group is a risk factor, none of the mothers of SGA infants had age $>40$ years. Contrary of general perception, in our study, the urban and rural mothers fared equally in the incidence of SGA fetus. Probably in the areas catered by this tertiary care hospital, the socioeconomic division does not exist.

Another important risk factor is the previous history of SGA birth. Compared to mothers with previous AGA births, mothers with previous SGA births had higher risk (RR - 3.9, $95 \%$ CI $3.4-4.6){ }^{11}$. In our study, the odds ratio was very high (OR 36, 95\% CI 4 - 310). Such a wide confidence interval was probably due to small size. It's important to educate women about adequate spacing and regarding the risk of subsequent SGA birth. If identified in $1^{\text {st }}$ trimester, such high-risk pregnancies should be referred to expert tertiary care center for management.

Anthropometric measures of weight and height were significantly low in mothers of SGA infants in comparison with controls. Since both were lower than controls, BMI as a ratio was not different between the two groups. In a study done in Brazil, 2200 mother-child pairs were assessed for relationship with SGA status. Short stature of mother and underweight status had odds of 3 and 2.3 respectively for the development of SGA infant ${ }^{12}$. In our study, maternal height, pre-pregnancy weight and weight gain during pregnancy had correlation coefficient of $0.36,0.27$ and 0.37 respectively. Moreover, while height was not a significant influencer, prepregnancy weight influenced the infant's birth weight every kilogram gain in weight by the mother reduced the risk of SGA infant by $0.8 \%$.

Inflammatory markers were hypothesized to play a role in predicting SGA fetuses. One study looked at the trend of change of CRP during pregnancy and concluded that the rate of change of CRP was negatively correlated with birth weight centiles $(\beta=-3.7,95 \% \text { CI }-5.5 \text { to }-2.1)^{13}$. In our study, probably due to very small numbers we were not able to show correlation between the third trimester CRP levels in the mothers of SGA infants with any outcomes.

There were several limitations in the current study and are enlisted below. The sample size of the study was calculated for prevalence study and not for case control 
The New Indian Journal of OBGYN. 2020 (July-December); 7(1)

study. Hence, some common correlations had not turned out to be significant. The definition of SGA was according to generally accepted $<10^{\text {th }}$ centile of estimated weight and not based much more stricter customized growth charts. PAPP-A and $\beta$-hCG levels were not significantly low in our study. Firstly, sample size was not calculated for these tests as a primary endpoint. Secondly, only a few mothers opted for this test in $1^{\text {st }}$ trimester and hence the numbers were small.

\section{Conclusion}

The prevalence of SGA fetuses in a tertiary care hospital in Kerala is $14 \%$. Mothers of SGA infants were leaner, stunted, had low hemoglobin, had less weight gain during pregnancy, delivered early and had higher cesarean rates. Health programs have to be strengthened to educate women regarding their adolescent health, as pre-pregnancy parameters were one of the most important determinants of SGA status of the fetus.

\section{Conflict of interest: None. Disclaimer: Nil.}

\section{References}

1. Nardozza LM, Caetano AC, Zamarian AC, Mazzola JB, Silva CP, Marcal VM, et al. Fetal growth restriction: current knowledge. Arch Gynecol Obstet. 2017; 295(5):1061-77.

2. Pallotto EK, Kilbride HW. Perinatal outcome and later implications of intrauterine growth restriction. Clin Obstet Gynecol. 2006; 49(2): 257-69.

3. Barker DJ. Adult consequences of fetal growth restriction. Clin Obstet Gynecol. 2006; 49(2): 270-83.

4. Sebastian T, Yadav B, Jeyaseelan L, Vijayaselvi R, Jose R. Small for gestational age births among South Indian women: temporal trend and risk factors from 1996 to 2010. BMC Pregnancy Childbirth. 2015;15:7.

5. Visentin S, Lapolla A, Londero AP, Cosma C, Dalfra M, Camerin M, et al. Adiponectin levels are reduced while markers of systemic inflammation and aortic remodelling are increased in intrauterine growth restricted mother-child couple. Biomed Res Int. 2014; 2014: 401595.

6. Lausten-Thomsen U, Olsen M, Greisen G, Schmiegelow $\mathrm{K}$. Inflammatory markers in umbilical cord blood from small-for-gestational-age newborns. Fetal Pediatr Pathol. 2014; 33(2): 114-8.

7. Buck Louis GM, Grewal J, Albert PS, Sciscione A, Wing DA, Grobman WA, et al. Racial/ethnic standards for fetal growth: the NICHD Fetal Growth Studies. Am J Obstet Gynecol. 2015; 213(4):449 e1- e41.

8. Kohn MA, Senyak J. Sample Size Calculators [Internet]. UCSF CTSI; [accessed on 13/11/2019]. Available at https://www.sample-size.net/

9. Kramer MS, Kakuma R. Energy and protein intake in pregnancy. Cochrane Database Syst Rev. 2003(4): CD000032.

10. Haider BA, Bhutta ZA. Multiple-micronutrient supplementation for women during pregnancy. Cochrane Database Syst Rev. 2017; 4: CD004905.

11. Okah FA, Cai J, Dew PC, Hoff GL. Risk factors for recurrent small-for-gestational-age birth. Am J Perinatol. 2010; 27(1): 1-7.

12. Britto RP, Florencio TM, Benedito Silva AA, Sesso R, Cavalcante JC, Sawaya AL. Influence of maternal height and weight on low birth weight: a cross-sectional study in poor communities of northeastern Brazil. PLoS One. 2013; 8(11): e80159.

13. de Oliveira LC, Franco-Sena AB, Farias DR, Rebelo F, Kac G. Maternal C-reactive protein concentrations during pregnancy and birth weight in a prospective cohort in Rio de Janeiro, Brazil. J Matern Fetal Neonatal Med. 2017; 30(19): 2346-53.

\footnotetext{
Subhadra Subramanian ${ }^{1}$, Remadevi $\mathrm{S}^{2}$, Karthik Subramaniam ${ }^{3}$

1,2 Department of Obstetrics and Gynacology, Lisie Hospital, Kochi, Kerala; India; ${ }^{3}$ Department of Endocrinology, Silverline Hospital, Kochi, Kerala, India.
} 